\title{
INHALT
}

Ludwig Nagl (Wien)

MEDIENPHILOSOPHIE - SYSTEMATISCH? EIN VORWORT

VII

Mike Sandbothe (Aalborg)

EINLEITUNG: WOZU SYSTEMATISCHE MEDIENPHILOSOPHIE?

XIII

\section{SINNLICHE WAHRNEHMUNGSMEDIEN}

Götz Großklaus (Karlsruhe)

MEDIENPHILOSOPHIE DES RAUMS ................................ 3

Ralf Beuthan (Jena)

MEDIENPHILOSOPHIE DER ZEIT .................................... 21

Michael Giesecke (Erfurt)

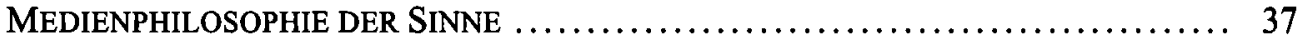

Barbara Becker (Paderborn)

MEDIENPHILOSOPHIE DER NAHSINNE $\ldots \ldots \ldots \ldots \ldots \ldots \ldots \ldots \ldots \ldots \ldots \ldots \ldots \ldots \ldots$

\section{SEMIOTISCHE INFORMATIONS- UND \\ KOMMUNIKATIONSMEDIEN}

Peter Janich (Marburg)

MEDIENPHILOSOPHIE DER KOMMUNIKATION $\ldots \ldots \ldots \ldots \ldots \ldots \ldots \ldots \ldots \ldots, 83$

Mathias Gutmann (Marburg)

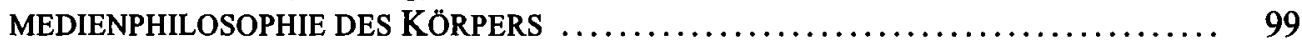

Dieter Mersch (Berlin)

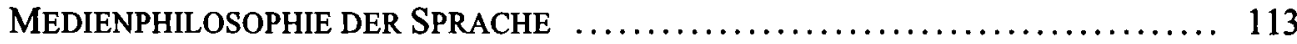

Christian Stetter (Aachen)

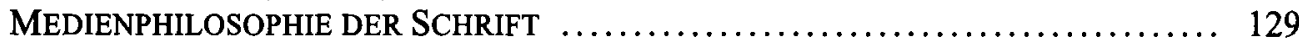

Lambert Wiesing (Jena)

MEDIENPHILOSOPHIE DES BILDES ............................... 147 
Matthias Vogel (Frankfurt a. M.)

MEDIENPHILOSOPHIE DER MUSIK

Gabriele Klein (Hamburg)

MEDIENPHILOSOPHIE DES TANZES

181

Dieter Teichert (Konstanz)

MEDIENPHILOSOPHIE DES THEATERS

\section{TECHNISCHE VERBREITUNGS-, VERARBEITUNGS- UND SPEICHERMEDIEN}

Sybille Krämer (Berlin)

MEDIENPHILOSOPHIE DER STIMME 221

Reinhard Margreiter (Innsbruck)

MEDIENPHILOSOPHIE DES BUCHDRUCKS

Bernd Stiegler (Frankfurt a. M.)

MEDIENPHILOSOPHIE DER PHOTOGRAPHIE

Alexander Roesler (Frankfurt a. M.)

MEDIENPHILOSOPHIE DES TELEFONS

Lorenz Engell (Weimar)

MEDIENPHILOSOPHIE DES FILMS 283

Christian Filk (Luzern)

MEDIENPHILOSOPHIE DES RADIOS

Stanley Cavell (Cambridge/Mass.)

MEDIENPHILOSOPHIE DES FERNSEHENS

Lutz Ellrich (Köln)

MEDIENPHILOSOPHIE DES COMPUTERS

Mark Poster (Irvine)

MEDIENPHILOSOPHIE DES INTERNET

Stefan Münker (Berlin)

MEDIENPHILOSOPHIE DER VIRTUAL REALITY 381

ZU DEN AUTORINNEN UND AUTOREN 397

PERSONENREGISTER 403 